\title{
The Rough Model Method (RMM) Application to The Computation of Normal Depth in Circular Conduit
}

\author{
Bachir Achour* and Sabah Sehtal
}

Research Laboratory in Subterranean and Surface Hydraulics (LARHYSS) University of Biskra, PO Box 145 RP 07000 Biskra, Algeria

\begin{abstract}
A new method is presented to compute the normal depth in circular conduit. This is the rough model method (RMM). It states that the linear dimension of a conduit or channel is equal to the linear dimension of a referential rough model corrected by the effect of a non-dimensional correction factor. The method is based on the Colebrook-White and Darcy-Weisbach relationships, applicable to the entire domain of turbulent flow. From the relationship governing the flow in the rough model, the normal depth in a circular conduit is explicitly deduced.
\end{abstract}

Keywords: Circular conduit, discharge, energy slope, normal depth, rough model method.

\section{INTRODUCTION}

The methods of calculating the uniform flow in conduits and channels are not numerous. Some of them are graphics and other iterative [1-3]. For circular and non-circular conduits, explicit calculation methods have been proposed in the past and one may quote as an example the method of Swamee and Swamee [4]. In this method, the design of noncircular conduits is based on a similar relation to that established for the circular pipe by Swamee and Jain [5]:

$D=k_{D} L$

With

$L=\left\{\varepsilon\left[Q^{2} /(g i)\right]^{4.8}+8 v Q^{9.4} /(g i)^{5.2}\right\}^{0.04}$

where $D$ is the vertical dimension of the conduit, $k_{D}$ is a constant depending on the shape of the conduit and $L$ is a linear dimension whose physical meaning has not been specified. Note that relation (1) is only applicable to the filling rate of $75 \%$. In Eq. (2), $Q$ is the discharge, $\varepsilon$ is the absolute roughness characterizing the state of the inner wall of the conduit, $g$ is the acceleration due to gravity, $i$ is the longitudinal slope of the conduit and $v$ is the Kinematic viscosity. In the study of Swamee and Swamee [4], an explicit approximate relation is proposed for estimating the filling rate in circular and non-circular conduits:

$\eta=k_{n 1}\left[k_{n 2}\left(Q_{\max } / Q-1\right)^{k_{n 3}}+1\right]^{-k_{n 3}}$

*Address correspondence to this author at the Research Laboratory in Subterranean and Surface Hydraulics, Biskra University, PO Box 145 RP 07000 Biskra Algeria; Tel: 00213557263947; Fax:0021333742481;

E-mail: bachir.achour@larhyss.net where $\eta=y_{n} / D, y_{n}$ is the normal depth, $D$ is the diameter of the conduit, $k_{n 1}, k_{n 2}$ and $k_{n 3}$ are constant values which depend on the shape of the geometric profile of the considered conduit. Note that the application of Eq. (3) requires the determination of the maximum discharge whose expression is established on the basis of statistical analysis of several curves. According to the authors, Eq. (3) is applicable for filling rate higher than $30 \%$ and it causes a maximum relative error of about $1 \%$.

The last known method for determining normal depth in circular conduit was published ten years ago [6]. Using Lagrange's theorem [7], the authors gave expression of the filling rate in the form of an infinite series, depending however on the coefficients of Chezy and Manning that are determined with great difficulty using iterative procedure, because these coefficients depend particularly on the filling rate $\eta$. The final result is obtained by truncating the series, leading to an approximate filling rate. As we can see below, the relationship determining the filling rate $\eta$ is not easy to handle for the use of the engineer. When Chezy's equation is used, filling rate $\eta$ is given as:

$$
\begin{aligned}
& \eta=\frac{1}{2}-\frac{1}{2} \cos \left(\frac{64 \pi}{3} M_{D}^{2}\right)^{1 / 3}+ \\
& \left(\frac{1}{4}-\frac{M_{D}^{2 / 3}}{3^{5 / 6} \pi^{2 / 3}}\right) \sin \left(\frac{128 \pi}{3} M_{D}^{2}\right)^{1 / 3}+\ldots
\end{aligned}
$$

where $M_{D}=Q /\left(C D^{2} \sqrt{D i}\right)$ and $C$ is Chezy's coefficient.

When Manning's equation is used, the filling rate $\eta$ is expressed as: 


$$
\begin{aligned}
& \eta=\frac{1}{2}-\frac{1}{2} \cos \left(\frac{32 \pi}{3} N_{D}^{3 / 2}\right)^{2 / 5}+ \\
& {\left[\frac{1}{4}-\frac{2}{5}\left(\frac{3^{1 / 6} N_{D}}{\pi}\right)^{3 / 5}\right] \sin \left(\frac{64 \pi}{3} N_{D}^{3 / 2}\right)^{2 / 5}+\ldots}
\end{aligned}
$$

where $N_{D}=n Q /\left(D^{8 / 3} \sqrt{i}\right)$ and $n$ is Manning's coefficient.

In this study, a simple method is proposed for the design of conduits and canals as well as the determination of normal depth. It is based on fitting a single curve, that of a referential rough model having the same shape as of the considered conduit. All hydraulic flow characteristics in the considered conduit are directly derived from those of the referential rough model which are known characteristics. The calculation method is presented through practical examples applied to the circular conduit.

\section{LOCATION OF THE PROBLEM}

Normal depth is an important parameter in the design of the pipes and channels, as well as in the varied flows analysis. In the literature, the normal depth is referred to as $y_{n}$. Fig. (1) shows schematically the normal depth in a circular conduit of diameter $D$.

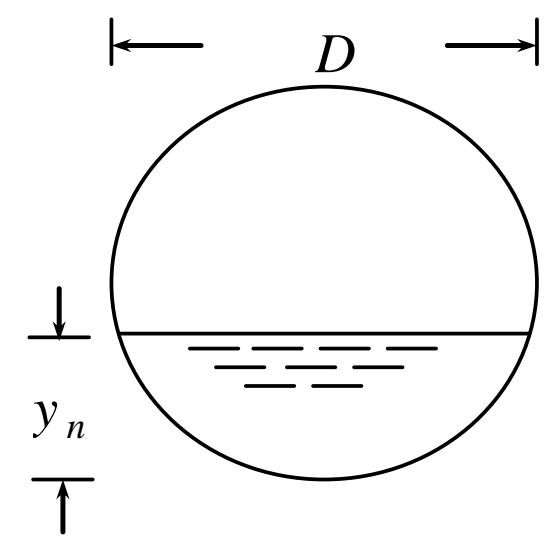

Fig. (1). Definition sketch of normal depth in circular conduit.

The calculation of normal depth is based primarily on the open channel resistance equations. The most commonly used equations in practice are namely the Darcy-Weisbach relationship, Chezy and Manning equations. The first one uses the friction factor as defined by Colebrook-White which is implicit. The solution involves many trials and tedious computations or laborious graphical procedure. The second and the third resistance equations use Chezy and Manning roughness coefficients that are not constant but vary depending especially on the filling rate and therefore on the normal depth. The calculation of these coefficients is done with a great difficulty using an inconvenient iterative process.

The problem is to compute normal depth using measurable data in practice such as the discharge $Q$, the slope $i$ of the conduit, the diameter $D$ of the conduit, the kinematic viscosity and mainly the absolute roughness $\varepsilon$ which reflects the state of the internal wall of the conduit. To solve the problem according to these data only, the RMM is the most appropriate method. This is what attempt to demonstrate the result of this study.

\section{FUNDAMENTAL RELATION OF THE RMM}

Consider on one hand a circular conduit of diameter $D$, flowing the discharge $Q$ of a fluid with $v$ as the kinematic viscosity, under a longitudinal slope $i$. The inner wall of the conduit is characterized by the absolute roughness $\varepsilon$. On the other hand, consider a referential rough model of the same shape defined by the diameter $\bar{D} \neq D$, the discharge $\bar{Q}=Q$, the kinematic viscosity $\bar{v}=v$ and a longitudinal slope $\bar{i}=i$ . The inner wall of the model is characterized by the relative roughness $\bar{\varepsilon} / \overline{D_{h}}=0.037$, where $\overline{D_{h}}$ is the hydraulic diameter. Due to the high relative roughness, the flow in the model is rough involving a friction factor $\bar{f}=1 / 16$ according to Colebrook-White relationship [9] for the Reynolds number $R \rightarrow \infty$. The diameters $D$ and $\bar{D}$ are not only different but are governed by the inequality $\bar{D}>D$. Between the diameters $D$ and $\bar{D}$, one can write the following equation:

$$
D=\psi \bar{D}
$$

where $\psi$ is the non-dimensional correction factor of linear dimensions such as $0<\psi<1$. Eq. (6) is the fundamental relation of the RMM. It can be generalized to all conduits and channels, writing that:

$$
L=\psi \bar{L}
$$

where $L$ is any linear dimension, such that the width of a rectangular channel, the hydraulic radius, the diameter of a circular pipe or the vertical dimension of a noncircular closed conduit etc... Eq. (7) can be written as $L^{2}=\psi^{2} \bar{L}^{2}$ and since $L^{2}$ and $\bar{L}^{2}$ are respectively proportional to the water areas $A$ and $\bar{A}$, then we can write :

$$
A=\psi^{2} \bar{A}
$$

\section{NON-DIMENSIONAL CORRECTION FACTOR OF LINEAR DIMENSIONS}

The Colebrook-White relationship [8] is applicable to any geometric profile of conduits or channels, since the form factor $\phi=R_{h, e} / R_{h}$ has a second-order effect [3], where $R_{h, e}$ is the effective hydraulic radius. Colebrook-White relationship [4] can then be written as:

$\frac{1}{\sqrt{f}}=-2 \log \left(\frac{\varepsilon / D_{h}}{3.7}+\frac{2.51}{R \sqrt{f}}\right)$

where $f$ is the friction factor and $R$ is the Reynolds number. According to Eq. (7), one can write the following equations: 
$D_{h}=\psi \overline{D_{h}}$

$R=\frac{4 Q}{P v}=\frac{4 Q}{\psi \bar{P} v}=\psi^{-1} \bar{R}$

Taking into account the Darcy-Weisbach relationship [8], the longitudinal slope of the conduit can be written as:

$i=\frac{f}{D_{h}} \frac{Q^{2}}{2 g A^{2}}=\bar{i}=\frac{\bar{f}}{\overline{D_{h}}} \frac{Q^{2}}{2 g \bar{A}^{2}}$

Hence:

$\frac{f}{D_{h} A^{2}}=\frac{1}{16 \overline{D_{h}} \overline{A^{2}}}$

Inserting equations (8) and (10) into Eq. (13), one can deduce:

$\psi=(16 f)^{1 / 5}$

Combining equations (8), (9), (11) and (14), leads to:

$\psi^{5 / 2} \log \left(\frac{\varepsilon / \overline{D_{h}}}{3.7 \psi}+\frac{10.04}{\psi^{3 / 2} \bar{R}}\right)=-2$

Eq. (15) is implicit towards the correction factor $\psi$ which must then be graphically estimated or computed with the aid of an iterative procedure. One way to avoid this is to use the following derived explicit relationship, obtained by a statistical analysis $[10,11]$ :

$\psi \cong 1.35\left[-\log \left(\frac{\varepsilon / \overline{D_{h}}}{4.75}+\frac{8.5}{\bar{R}}\right)\right]^{-2 / 5}$

A comparison was made between equations (15) and (16), varying $\varepsilon / \overline{D_{h}}$ from 0 to 0.02 . It revealed that maximum deviation is less than $0.4 \%$ for $\bar{R} \geq 2200$ corresponding to $R \geq 2300$. Eq. (16) is applicable in all the field of turbulent flow, corresponding to $R \geq 2300$ and to the wide range $0 \leq \varepsilon / D_{h} \leq 0.05$. Eq. (16) is applicable to any form of conduits and channels. It is valid for any filling rate and shape parameter channels. It is more general than is Eq. (1).

\section{NORMAL DEPTH COMPUTATION IN CIRCULAR CONDUIT USING RMM}

For the rough model, Chezy's equation can be written as:

$$
\bar{Q}=Q=\bar{C} \bar{A} \sqrt{\overline{R_{h}} i}
$$

Since $\bar{f}=1 / 16$, one can write $\bar{C}=\sqrt{8 g / \bar{f}}=8 \sqrt{2 g}$. The wetted area $\bar{A}$ is governed by the following relation:

$$
\bar{A}=\frac{\bar{D}^{2}}{4}\left[\cos ^{-1}(1-2 \bar{\eta})-2(1-2 \bar{\eta}) \sqrt{\bar{\eta}(1-\bar{\eta})}\right]
$$

Where $\bar{\eta}=\overline{y_{n}} / \bar{D}$ is the filling rate in the rough model and $\overline{y_{n}}$ is the normal depth. Eq. (18) can be written as:

$\bar{A}=\frac{\bar{D}^{2}}{4} \sigma(\bar{\eta}) \varphi(\bar{\eta})$

where :

$\sigma(\bar{\eta})=\cos ^{-1}(1-2 \bar{\eta})$

$\varphi(\bar{\eta})=1-\frac{2(1-2 \bar{\eta}) \sqrt{\bar{\eta}(1-\bar{\eta})}}{\cos ^{-1}(1-2 \bar{\eta})}$

The wetted perimeter $\bar{P}$ is given by:

$\bar{P}=\bar{D} \cos ^{-1}(1-2 \bar{\eta})$

or :

$\bar{P}=\bar{D} \sigma(\bar{\eta})$

The hydraulic radius $\overline{R_{h}}=\bar{A} / \bar{P}$ is then:

$\overline{R_{h}}=\frac{\bar{D}}{4} \varphi(\bar{\eta})$

Taking into account Eqs. (19) and (24) and the fact that $\bar{C}=8 \sqrt{2 g}$, Eq. (17) is written as:

$\bar{Q}=Q=\sqrt{2} \sqrt{g i \bar{D}^{5}} \sigma(\bar{\eta})[\varphi(\bar{\eta})]^{3 / 2}$

The relative conductivity $\bar{Q}^{*}=\bar{Q} / \sqrt{g i \bar{D}^{5}}$ is then:

$\bar{Q}^{*}=\sqrt{2} \sigma(\bar{\eta})[\varphi(\bar{\eta})]^{3 / 2}$

The relative conductivity $\bar{Q}^{*}$ in the rough model is thus function of the filling rate $\bar{\eta}$. Eq. (26) was plotted in Fig. (2). It shows that the relative conductivity in the rough

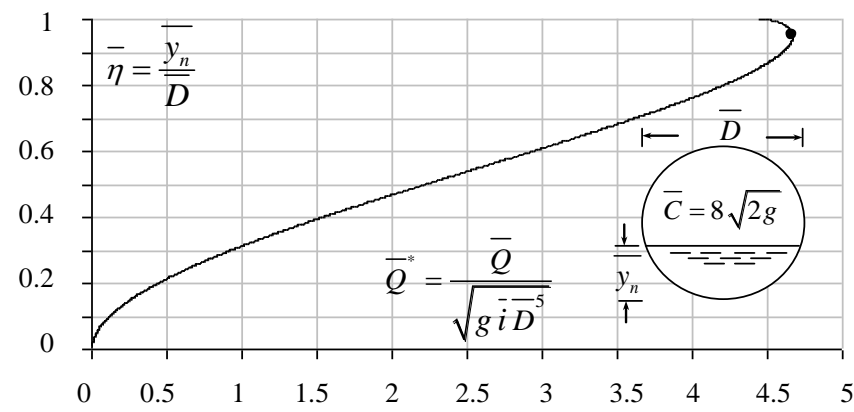

Fig. (2). Plot of Eq. (26).

(•) Maximum relative conductivity corresponding to $\bar{\eta}=0.95$ 
model begins with an ascending phase, then reaches a maximum and finally undergoes a decreasing phase beyond this maximum. The calculation showed that the maximum relative conductivity is achieved for the filling rate $\bar{\eta}=0.95$.

In the wide range $0.15 \leq \bar{\eta} \leq 0.85$, corresponding to $0.255 \leq \bar{Q}^{*} \leq 4.433$, a special study of Eq. (26) has shown that the filling rate $\bar{\eta}$ could be expressed, with a maximum relative deviation less than $0.3 \%$ only, by the following approximate relation:

$\sin \left(\frac{5 \pi}{11} \bar{\eta}\right)=0.432 \bar{Q}^{* 0.519}$

Consider a referential rough model having a diameter $\bar{D}$ equal to that of the full-model state corresponding to $\bar{\eta}=1$; Eqs. (20) and (21) give respectively $\sigma(\bar{\eta})=\pi$ and $\varphi(\bar{\eta})=1$. As a result, Eq. (26) leads to $\bar{Q}^{*}=\pi \sqrt{2}$. For this value, Fig. (2) indicates a filling rate $\bar{\eta} \approx 0.852$. We thus obtain a rough model with a diameter equal to that of the full-model state, characterized by the filling rate $\bar{\eta} \approx 0.852$. For this filling rate, Eqs. (23) and (24) allow to write respectively that:

$\bar{P}=2.352 \bar{D}$

$\overline{D_{h}}=1.2126 \bar{D}$

The rough full-model diameter is obtained for the relative conductivity $Q=\pi \sqrt{2}$, applying what follows:

$\bar{D}=(\pi \sqrt{2})^{-0.4}\left(\frac{Q}{\sqrt{g i}}\right)^{0.4}$

For the data of the problem, namely $Q, D, i, \varepsilon$ and $v$, Eqs. (28), (29) and (30) are used to calculate respectively the characteristics $\bar{D}, \bar{P}$ and $\overline{D_{h}}$ of the referential rough model and hence the value of the Reynolds number $\bar{R}=4 Q /(\bar{P} v)$. For the calculated values of $\overline{D_{h}}$ and $\bar{R}$, Eq. (16) allows to compute explicitly the non-dimensional correction factor of linear dimensions $\psi$.

If we affect to the referential rough model the new linear dimension $D / \psi$, according to Eq. (6), the filling rate $\bar{\eta}$ in the rough model would be equal to the filling rate $\eta$ in the considered conduit. This filling rate is given by Eq. (27) for the relative conductivity $\bar{Q}^{*}=Q / \sqrt{g i(D / \psi)^{5}}$. The required normal depth is then $y_{n}=\eta D$.

\section{EXAMPLE 1}

Compute the relative normal depth in circular conduit for the following data, using the RMM:

$$
Q=3 m^{3} / s, D=2 m, i=5 \times 10^{-4}, \varepsilon \rightarrow 0, v=10^{-6} \mathrm{~m}^{2} / \mathrm{s}
$$

Note that the resistance coefficient $C$ of Chezy or $n$ of Manning is not required.

Calculate the diameter $\bar{D}$ of the full rough model using Eq. (30). Hence:

$$
\begin{aligned}
\bar{D} & =(\pi \sqrt{2})^{-0.4}\left(\frac{Q}{\sqrt{g i}}\right)^{0.4} \\
& =(\pi \times \sqrt{2})^{-0.4}\left(\frac{3}{\sqrt{9.81 \times 5 \times 10^{-4}}}\right)^{0.4} \approx 2.475 \mathrm{~m}
\end{aligned}
$$

According to Eq. (28), the wetted perimeter $\bar{P}$ is :

$$
\bar{P}=2.352 \bar{D}=2.352 \times 2.475=5.8212 \mathrm{~m}
$$

The Reynolds number $\bar{R}$ is :

$$
\bar{R}=4 Q /(\bar{P} v)=4 \times 3 /\left(5.8212 \times 10^{-6}\right)=2061430.63
$$

1. According to Eq. (16), the non-dimensional correction factor of linear dimensions $\psi$ is then :

$$
\begin{aligned}
& \psi \cong 1.35\left[-\log \left(\frac{8.5}{\bar{R}}\right)\right]^{-2 / 5} \\
& =1.35 \times\left[-\log \left(\frac{8.5}{2061430.63}\right)\right]^{-2 / 5}=0.6884
\end{aligned}
$$

Compute the relative conductivity $\bar{Q}^{*}$ for the new linear dimension :

$$
\bar{D}=D / \psi=2 / 0.6884=2.905 m \text {. }
$$

Hence:

$$
\begin{aligned}
\bar{Q}^{*} & =Q / \sqrt{g i(D / \psi)^{5}} \\
& =3 / \sqrt{9.81 \times 5 \times 10^{-4} \times 2.905^{5}}=2.978
\end{aligned}
$$

According to Eq. (27), the filling rate is thus :

$$
\begin{aligned}
\bar{\eta} & =\eta=\frac{11}{5 \pi} \sin ^{-1}\left(0.432 \bar{Q}^{* 0.519}\right) \\
& =\frac{11}{5 \times \pi} \times \sin ^{-1}\left(0.432 \times 2.978^{0.519}\right) \approx 0.605
\end{aligned}
$$

2. The required value of the normal depth is then :

$y_{n}=\eta D=0.605 \times 2=1.21 \mathrm{~m}$ 
3. Check the calculations by determining the discharge $Q$ by the use of Eq. (17). For the calculated filling rate $\bar{\eta}=\eta=0.605$, Eqs. (20) and (21) give respectively:

$$
\begin{aligned}
\sigma(\bar{\eta}) & =\cos ^{-1}(1-2 \bar{\eta})=\cos ^{-1}(1-2 \times 0.605)=1.7824 \\
\varphi(\bar{\eta}) & =1-\frac{2(1-2 \bar{\eta}) \sqrt{\bar{\eta}(1-\bar{\eta})}}{\cos ^{-1}(1-2 \bar{\eta})} \\
& =1-\frac{2 \times(1-2 \times 0.605) \times \sqrt{0.605 \times(1-0.605)}}{\cos ^{-1}(1-2 \times 0.605)} \\
& =1.1152
\end{aligned}
$$

As a result, Eq. (19) gives the wetted area $\bar{A}$ as:

$$
\begin{aligned}
\bar{A} & =\frac{\bar{D}^{2}}{4} \sigma(\bar{\eta}) \varphi(\bar{\eta})=\frac{2.905^{2}}{4} \times 1.7824 \times 1.1152 \\
& =4.1936 \mathrm{~m}^{2}
\end{aligned}
$$

According to Eq. (24), the hydraulic radius $\overline{R_{h}}$ is then:

$\overline{R_{h}}=\frac{\bar{D}}{4} \varphi(\bar{\eta})=\frac{2.905}{4} \times 1.1152=0.81 \mathrm{~m}$

Finally, Eq. (17) gives the discharge $Q$ as:

$$
\begin{aligned}
\bar{Q} & =Q=\bar{C} \bar{A} \sqrt{\overline{R_{h}} i} \\
& =8 \times \sqrt{2 \times 9.81} \times 4.1936 \times \sqrt{0.81 \times 5 \times 10^{-4}} \\
& =2.99 \mathrm{~m}^{3} / \mathrm{s} \approx 3 \mathrm{~m}^{3} / \mathrm{s}
\end{aligned}
$$

As one can see, the calculated discharge corresponds to the discharge given in the problem statement, confirming the validity of the calculations.

The calculations could be also checked by the general relationship of the discharge [12].

\section{EXAMPLE 2}

Let us propose another example of the normal depth calculation in circular conduit for a positive absolute roughness, in order to confirm the reliability of the RMM. Consider the following data:

$Q=1 \mathrm{~m}^{3} / \mathrm{s}, \quad D=1.7 \mathrm{~m}, \quad i=4 \times 10^{-4}, \quad \varepsilon=0.001 \mathrm{~m}$, $v=10^{-6} \mathrm{~m}^{2} / \mathrm{s}$

1. Using Eq. (30), the diameter $\bar{D}$ of full rough model is :

$$
\begin{aligned}
\bar{D} & =(\pi \sqrt{2})^{-0.4}\left(\frac{Q}{\sqrt{g i}}\right)^{0.4} \\
& =(\pi \times \sqrt{2})^{-0.4}\left(\frac{1}{\sqrt{9.81 \times 4 \times 10^{-4}}}\right)^{0.4} \approx 1.668 m
\end{aligned}
$$

2. According to Eq. (28), the wetted perimeter $\bar{P}$ is :

$\bar{P}=2.352 \bar{D}=2.352 \times 1.668=3.923 \mathrm{~m}$

3. Using Eq. (29), the hydraulic diameter $\overline{D_{h}}$ is then:

$\overline{D_{h}}=1.2126 \bar{D}=1.2126 \times 1.668 \cong 2.0226 \mathrm{~m}$

4. With the calculated value of $\bar{P}$, Reynolds number $\bar{R}$ in the rough model is:

$\bar{R}=4 Q /(\bar{P} v)=4 \times 1 /\left(3.923 \times 10^{-6}\right)=1019610.79$

5. Using Eq. (16), the non-dimensional correction factor of linear dimension $\psi$ is as:

$\psi=1.35\left[-\log \left(\frac{\varepsilon / \overline{D_{h}}}{4.75}+\frac{8.5}{\bar{R}}\right)\right]^{-2 / 5}$

$\psi=1.35 \times\left[-\log \left(\frac{0.001 / 2.0226}{4.75}+\frac{8.5}{1019610.79}\right)\right]^{-2 / 5}$

$=0.77935$

6. Assign to the rough model the following new linear dimension, according to Eq. (6):

$\bar{D}=D / \psi=1.7 / 0.77935=2.1813 \mathrm{~m}$ then:

The corresponding value of the relative conductivity is

$$
\begin{aligned}
\bar{Q}^{*} & =Q / \sqrt{g i(D / \psi)^{5}} \\
& =1 / \sqrt{9.81 \times 4 \times 10^{-4} \times 2.1813^{5}}=2.27167
\end{aligned}
$$

7. The required value of the filling rate $\eta$ is given by Eq. (27) as:

$$
\begin{aligned}
\bar{\eta} & =\eta=\frac{11}{5 \pi} \sin ^{-1}\left(0.432 \bar{Q}^{* 0.519}\right) \\
& =\frac{11}{5 \times \pi} \times \sin ^{-1}\left(0.432 \times 2.27167^{0.519}\right) \approx 0.506
\end{aligned}
$$

8. Finally, the normal depth is:

$$
y_{n}=\eta D=0.506 \times 1.7=0.86 m
$$

9. Verify the validity of the calculations by determining the discharge $Q$ using Eq. (17). Compute first $\sigma \bar{\eta})$ and $\varphi(\bar{\eta})$ using Eqs. (20) and (21) respectively. Hence:

$$
\sigma(\bar{\eta})=\cos ^{-1}(1-2 \bar{\eta})=\cos ^{-1}(1-2 \times 0.506)=1.58285
$$

$$
\varphi(\bar{\eta})=1-\frac{2(1-2 \bar{\eta}) \sqrt{\bar{\eta}(1-\bar{\eta})}}{\cos ^{-1}(1-2 \bar{\eta})}
$$




$$
\begin{aligned}
& =1-\frac{2 \times(1-2 \times 0.506) \times \sqrt{0.506 \times(1-0.506)}}{\cos ^{-1}(1-2 \times 0.506)} \\
& =1.00762
\end{aligned}
$$

According to Eq. (19), the water area $\bar{A}$ is:

$$
\begin{aligned}
\bar{A} & =\frac{\bar{D}^{2}}{4} \sigma(\bar{\eta}) \varphi(\bar{\eta})=\frac{2.1813^{2}}{4} \times 1.58285 \times 1.00762 \\
& =1.8972 \mathrm{~m}^{2}
\end{aligned}
$$

According to Eq. (24), the hydraulic radius $\overline{R_{h}}$ is as:

$$
\overline{R_{h}}=\frac{\bar{D}}{4} \varphi(\bar{\eta})=\frac{2.1813}{4} \times 1.00762=0.5495 \mathrm{~m}
$$

Using Eq. (17), the discharge $Q$ is then:

$$
\begin{aligned}
\bar{Q} & =Q=\bar{C} \bar{A} \sqrt{\overline{R_{h}} i} \\
& =8 \times \sqrt{2 \times 9.81} \times 1.8972 \times \sqrt{0.5495 \times 4 \times 10^{-4}} \\
& =0.9967 \mathrm{~m}^{3} / \mathrm{s} \approx 1 \mathrm{~m}^{3} / \mathrm{s}
\end{aligned}
$$

This second example shows once again that the calculated discharge is equal to the discharge given in the problem statement, confirming the validity of the calculations.

\section{CONCLUSION}

A new computation method of normal depth in circular conduit is presented. This is the rough model method (RMM) which states that any linear dimension of a conduit or channel is equal to the linear dimension of a referential rough model, corrected by the effect of a dimensionless factor.

The method is based on Colebrook-White and DarcyWeisbach relationships and is, therefore, valid throughout the field of turbulent flow.

The application of the method for the determination of normal depth in a circular conduit is presented. It leads to the establishment of an excellent approximate equation expressing the filling rate, depending on the relative conductivity in the referential rough model. This is characterized by a diameter equal to that of the full-model state and by a filling rate of 0.852 . The obtained relationship allows us to deduce explicitly the normal depth in the considered circular conduit, using the fundamental relationship of the RMM. Practical examples are taken to explain the process of calculation. The proposed method does not require the coefficients of Chezy and Manning, unlike current methods of calculation. The theoretical development as well as the calculation examples we proposed show no restriction in the application of the rough model method.

\section{NOTATION}

$A=$ Water area
$C=$ Chezy's coefficient
$D=$ Diameter of the conduit
$D_{h}=$ Hydraulic diameter
$f=$ Friction factor
$g=$ Acceleration due to gravity
$i=$ Slope of the conduit
$L=$ Linear dimension
$P=$ Wetted perimeter
$Q=$ Discharge
$Q^{*}=$ Relative conductivity
$R=$ Reynolds number
$R_{h}=$ Hydraulic radius
$y_{n}=$ Normal depth
$\varepsilon=$ Absolute roughness
$\eta=$ Filling rate equal to $y_{n} / D$
$\psi=$ Non-dimensional factor
$v=$ kinematic viscosity

\section{CONFLICT OF INTEREST}

The authors confirm that this article content has no conflicts of interest.

\section{ACKNOWLEDGEMENTS}

None declared.

\section{REFERENCES}

[1] V.T. Chow, Ed., Open-Channel Hydraulics. McGraw Hill: New York, 1973.

[2] R.H. French, Ed., Open Channel Hydraulics. McGraw Hill: New York, 1986.

[3] R.O. Sinniger and W.H. Hager, Ed., Constructions hydrauliques. Presses Polytechniques Romandes: Suisse, 1989.

[4] P.K. Swamee and N. Swamee, "Design of noncircular sewer sections", J. Hydraulic Res., vol. 46, no. 2, pp. 277-281, 2008.

[5] P.K. Swamee and A.K. Jain, "Explicit equations for pipe-flow problems", J. Hydraulic Eng., ASCE, vol. 102 (HY5), no. 657-664, (HY11), pp. 1707-1709, 1976.

[6] P.K. Swamee and P.N. Rathie, "Exact solutions for normal depth problem", J. Hydraulic Res., vol. 42, no. 5, pp. 541-547, 2004.

[7] E.T. Whittaker and G.N. Watson, A Course of Modern Analysis. Cambridge University Press, Cambridge: UK, 1965.

[8] H. Darcy , Sur les recherches expérimentales relatives au mouvement des eaux dans les tuyaux, Comptes rendus des séances de l'Académie des Sciences, no. 38, pp. 1109-1121, 1854.

[9] C.F. Colebrook, "Turbulent flow in pipes, with particular reference to the transaction region between the smooth and rough pipe laws", J. Inst. Civil Eng., vol. 11, pp. 133-156, 1939.

[10] B. Achour and A. Bedjaoui, "Turbulent pipe-flow computation using the rough model method (RMM)", J. Civil Eng. Sci., vol. 1, no. 1, pp. 36-41, 2012. 
[11] B. Achour, "Design of pressurized vaulted rectangular conduits using the rough model method", Adv. Mat. Res., vol. 779-780, 414419, pp. , 2013.
[12] B. Achour and A. Bedjaoui, "Discussion. Exact solutions for normal depth problem", J. Hydraulic Res., vol. 44, no. 5, pp. 715717, 2006.

Received: January 09, 2014

Revised: March 24, 2014

Accepted: March 25, 2014

(c) Achour and Sehtal; Licensee Bentham Open.

This is an open access article licensed under the terms of the Creative Commons Attribution Non-Commercial License (http://creativecommons.org/licenses/ by-nc/3.0/) which permits unrestricted, non-commercial use, distribution and reproduction in any medium, provided the work is properly cited. 